Publ. RIMS, Kyoto Univ.

18 (1982), 413-426

\title{
Poisson's Summation Formula and Hamburger's Theorem
}

\author{
By \\ Leon EHRENPREIS* and Takahiro KAWAI**
}

Poisson's summation formula tells us that the Fourier transform of the sum of Dirac's $\delta$-functions supported by the integral points is again the sum of Dirac's $\delta$-functions supported by the integral points. In this paper we first consider the converse problem, that is, we characterize a distribution which is a sum of distributions supported by the integral points and whose Fourier transform is again of the same form. Using this result we give another proof of the classical theorem of Hamburger on the characterization of the zata function of Riemann. We also show a generalization of the result to the zeta function associated to the imaginary quadratic field $Q(\sqrt{-1})$.

In what follows, we use the notation $\mathscr{F}(f)$ or $\hat{f}$ to denote the Fourier transform of $f$ normalized in the form $\int f(x) \exp (2 \pi \sqrt{-1}\langle x, \xi\rangle) d x$. We denote by $D_{x}^{\alpha}$ (resp., $\left.x^{\alpha}\right) \quad\left(\alpha=\left(\alpha_{1}, \cdots, \alpha_{m}\right) \in \mathbb{Z}_{+}^{m}\right)$ the differential operator $\partial^{|\alpha|} / \partial x_{1}^{\alpha_{1}} \cdots \partial x_{m}^{\alpha_{m}}$ (resp., the monomial $x_{1}^{\alpha_{1}} \cdots x_{m}^{\alpha_{m}}$ ), where $\mathbb{Z}_{+}^{m}$ denotes the set of $m$-tuple of non-negative integers and $|\alpha|=\sum_{j=1}^{m} \alpha_{j}$. We denote Dirac's $\delta$-function supported at $x=n$ by $\delta(x-n)$ and its derivative $D_{x}^{\alpha} \delta(x-n)$ by $\delta^{(\alpha)}(x-n)$.

Now the first result is stated as follows:

Theorem 1. Let $N$ and $m$ be strictly positive integers. Let $a_{\alpha, n}$ and $b_{\beta, \nu}\left(\alpha, \beta \in \mathbb{Z}_{+}^{m},|\alpha|,|\beta|<N, n, \nu \in \mathbb{Z}^{m}\right)$ be complex numbers which satisfy

$$
\left|a_{\alpha, n}\right| \leqq C|n|^{M}
$$

Received July 8, 1981.

* Yeshiva University, New York, 10033, U.S.A.

** Research Institute for Mathematical Sciences, Kyoto University, Kyoto 606, Japan. 
and

$$
\left|b_{\beta, \nu}\right| \leqq C|\nu|^{M}
$$

for some constants $C$ and $M$. Suppose that

$$
\mathscr{I}\left(\sum_{\alpha, n} a_{\alpha, n} \delta^{(\alpha)}(x-n)\right)=\sum_{\beta, \nu} b_{\beta, \nu} \delta^{(\beta)}(\xi-\nu) .
$$

Then there exists a linear differential operator $P\left(x, D_{x}\right)$ with poly. nomial coefficients such that

$$
\sum_{\alpha, n} a_{\alpha, n} \delta^{(\alpha)}(x-n)=P\left(x, D_{x}\right)\left(\sum_{n \in Z^{m}} \delta(x-n)\right) .
$$

Furthermore the order of $P$ is less than $N$ and the degree of each coefficient of $P$ is less than $N$.

Remark. Conditions (1) and (2) guarantee that both sides of (3) are well-defined tempered distributions.

Proof of Theorem 1. Let $S_{l}(\xi)$ denote the distribution $\mathscr{E}^{-1}\left(\sin \left(\pi x_{l}\right)\right)$, i.e.,

$$
\frac{\sqrt{-1}}{2}\left(\delta\left(\xi_{l}+\frac{1}{2}\right)-\delta\left(\xi_{l}-\frac{1}{2}\right)\right) \prod_{l^{\prime} \neq l} \delta\left(\xi_{l^{\prime}}\right)
$$

and let $T_{l}(\xi)$ denote the $N$-th interated convolution product of $S_{l}$, i.e.,

$$
\left(\frac{\sqrt{-1}}{2}\right)^{N}\left(\sum_{k=0}^{N} \frac{N !(-1)^{k}}{k !(N-k) !} \delta\left(\xi_{l}+\frac{N}{2}-k\right) \prod_{l^{\prime} \neq l} \delta\left(\xi_{l^{\prime}}\right)\right) .
$$

Since $|\alpha|<N$,

$$
\left[\sin \left(\pi x_{l}\right)\right]^{N}\left(\sum_{\alpha, n} a_{\alpha, n} \hat{\delta}^{(\alpha)}(x-n)\right)=0
$$

holds. Hence we have

$$
T_{l} *\left(\sum_{\beta, \nu} b_{\beta, \nu} \delta^{(\beta)}(\xi-\nu)\right)=0 .
$$

Now let $\Delta_{\beta}$ denote $\sum_{\nu} b_{\beta, \nu} \delta(\xi-\nu)$. Since $T_{l}$ is a sum of finitely many $\delta$-functions, $T_{l} *\left(D^{\beta} \Delta_{\beta}\right)$ is again of the form $D^{\beta}\left(\sum_{\nu} c_{\beta, \nu, l} \delta(\xi-\nu)\right)$ with $c_{\beta, \nu, l}$ in $\boldsymbol{C}$. Hence (5) implies

$$
T_{l} * D^{\beta} \Delta_{\beta}=0
$$

for any $l$ and $\beta$. Then, by the inverse Fourier transformation, we get 
(6) $\beta, l$

$$
(2 \pi \sqrt{-1} x)^{\beta} \mathscr{E}^{-1}\left(T_{l}\right) \mathscr{F}^{-1} \Delta_{\beta}=0 .
$$

Since $\mathscr{L}^{-1}\left(T_{l}\right)=\sin \left(\pi x_{l}\right)^{N}$ holds by definition, $(6)_{\beta, l}$ entails that $\operatorname{supp}\left(\mathscr{F}^{-1} \Delta_{\beta}\right)$ is contained in $\left\{x=\left(x_{1}, \cdots, x_{m}\right) \in \mathbb{R}^{m} ; x_{l} \in \mathbb{Z}\right\}$. Since this is true for each $l, \operatorname{supp}\left(\mathscr{F}^{-1} \Delta_{\beta}\right)$ is contained in $\left\{x \in \mathbb{Z}^{m}\right\}$. In particular,

$$
\mathscr{E}^{-1} \Delta_{\beta}=\sum_{|r| \leqq N_{\beta}} d_{\beta, \gamma} \delta^{(r)}(x)
$$

holds in a neighborhood of the origin, for some integer $N_{\beta}$ and constants $d_{\beta, r}$. On the other hand, we have

$$
\mathscr{F}^{-1} \Delta_{\beta}=\sum_{\nu} b_{\beta, \nu} \exp (-2 \pi \sqrt{-1}\langle x, \nu\rangle) .
$$

Hence $\mathscr{F}^{-1} \Delta_{\beta}$ is periodic. Therefore (7) entails

$$
\begin{aligned}
\mathscr{F}^{-1} \Delta_{\beta} & =\sum_{\substack{|r| \leq N_{\beta} \\
n \in \mathbb{Z}^{m}}} d_{\beta, \tau} \delta^{(r)}(x-n) \\
& =\sum_{|r| \leqq N_{\beta}} d_{\beta, \tau} D_{x}^{r}\left(\sum_{n \in \mathbb{Z}^{m}} \delta(x-n)\right) .
\end{aligned}
$$

Then, by Poisson's summation formula, we obtain

$$
\mathscr{F}^{-1} \Delta_{\beta}=\sum_{|\gamma| \varliminf_{\beta}} d_{\beta, \gamma} D_{x}^{r}\left(\mathscr{F}^{-1} \sum_{\nu \in \mathbb{Z}^{m}} \delta(\xi-\nu)\right) .
$$

Hence we have

$$
\begin{aligned}
\Delta_{\beta} & =\sum_{|r| \leq N_{\beta}} d_{\beta, r}\left(-2 \pi \sqrt{-1 \xi)^{r}}\left(\sum_{\nu \in \mathbb{Z}^{m}} \delta(\xi-\nu)\right)\right. \\
& =\sum_{\substack{|r| \leq N_{\beta} \\
\nu \in \mathbb{Z}^{m}}} d_{\beta, r}(-2 \pi \sqrt{-1} \nu)^{r} \delta(\xi-\nu) .
\end{aligned}
$$

This implies

$$
b_{\beta, \nu}=\sum_{|r| \leqq N_{\beta}} d_{\beta, r}(-2 \pi \sqrt{-1} \nu)^{r},
$$

that is, $b_{\beta, \nu}$ is a polynomial $B_{\beta}(\nu)$ of $\nu$ whose degree is $\leqq N_{\beta}$.

$\mathrm{By}$ applying the inverse Fourier transformation to (3) we see that $a_{\alpha, n}$ is also a polynomial $A_{\alpha}(n)$ of $n$ whose degree is $N_{\alpha}$. In what follows $N^{\prime}$ denotes $\max _{\alpha, \beta}\left\{N_{\alpha}, N_{\beta}\right\}$. Thus (3) takes the form

$$
\mathscr{F}\left(\sum_{\alpha, n} A_{\alpha}(n) D_{x}^{\alpha} \delta(x-n)\right)=\sum_{\beta, \nu} B_{\beta}(\nu) D_{\xi}^{\beta} \delta(\xi-\nu) .
$$

Since

$$
\begin{aligned}
\sum_{\alpha, n} A_{\alpha}(n) D_{x}^{\alpha} \delta(x-n) & =\sum_{\alpha, n} D_{x}^{\alpha} A_{\alpha}(n) \delta(x-n) \\
& =\sum_{\alpha, n} D_{x}^{\alpha}\left[A_{\alpha}(x) \delta(x-n)\right]
\end{aligned}
$$


holds, (9) combined with Poisson's summation formula entails

$$
\begin{aligned}
& \sum_{\alpha, n}(-2 \pi \sqrt{-1 \xi})^{\alpha}\left(A_{\alpha}\left(D_{\xi} / 2 \pi \sqrt{-1}\right) \mathcal{F}(\delta(x-n))\right) \\
& =\sum_{\alpha}(-2 \pi \sqrt{-1} \xi)^{\alpha} A_{\alpha}\left(D_{\xi} / 2 \pi \sqrt{-1}\right)\left(\mathscr{E}\left(\sum_{n} \delta(x-n)\right)\right) \\
& =\sum_{\alpha}(-2 \pi \sqrt{-1 \xi})^{\alpha} A_{\alpha}\left(D_{\xi} / 2 \pi \sqrt{-1}\right)\left(\sum_{\nu} \delta(\xi-\nu)\right) \\
& =\sum_{\beta, \nu} D_{\xi}^{\beta}\left(B_{\beta}(\xi) \delta(\xi-\nu)\right) \text {. }
\end{aligned}
$$

Now we rewrite the operator $\sum_{\alpha}(-2 \pi \sqrt{-1} \xi)^{\alpha} A_{\alpha}\left(D_{\xi} / 2 \pi \sqrt{-1}\right)$ (resp., $\left.\sum_{\beta} D_{\xi}^{\beta} \cdot B_{\beta}(\xi)\right)$ in the form $\sum_{s, t \in \mathbb{Z}_{+}^{m}} a_{s, t} D_{\xi}^{s} \cdot \xi^{t}$ (resp., $\sum_{u, v \in \mathbb{Z}_{+}^{m}} b_{u, v} D_{\xi}^{u} \cdot \xi^{v}$ ) with $a_{s, t}$ and $b_{u, v}$ being complex numbers. Then it follows from the assumption that

$$
a_{s, t}=0 \quad \text { if } \quad|t| \geqq N
$$

and

$$
b_{u, v}=0 \quad \text { if } \quad|u| \geqq N .
$$

On the other hand, comparing the coefficients of $D_{\xi}^{w} \delta(\xi-\nu)$ in (10), we find

$$
\sum_{t} a_{w, l} \nu^{t}=\sum_{v} b_{w, v} \nu^{v}
$$

holds for every $w \in \boldsymbol{Z}_{+}^{m}$ and $\nu \in \boldsymbol{Z}^{m}$. Since $a_{w, t}$ (resp., $b_{w, v}$ ) vanishes if $|w|>N^{\prime}$ (resp., $\left.|v|>N^{\prime}\right)$, (13) implies that $a_{w, t}=b_{w, t}$ holds for every $(w, t) \in \boldsymbol{Z}_{+}^{n} \times \boldsymbol{Z}_{+}^{n}$. In particular, $a_{w, t}$ vanishes if $|w| \geqq N$. This means that the operator $\sum_{s, t} a_{s, t} D_{\xi}^{s} \cdot \xi^{t}$ is of order $<N$. Defining $P\left(x, D_{x}\right)$ by

$$
\sum_{\substack{s, t \in \mathbf{Z}_{+}^{m} \\|s|,|t|<N}} a_{s, t}(2 \pi \sqrt{-1} x)^{s}\left(-D_{x} / 2 \pi \sqrt{-1}\right)^{t},
$$

we obtained the required operator $P$.

Q.E.D.

We now discuss how Theorem 1 is related to the classical theorem of Hamburger [3] on the characterization of the zeta function $\zeta(s)$ of Riemann.

Let $\left\{a_{n}\right\}_{n=1,2, \ldots}$ and $\left\{b_{\nu}\right\}_{\nu=1,2, \ldots}$ be sequences of complex numbers which satisfy the following condition.

(14) There exist constants $C$ and $M$ for which 


$$
\left|a_{n}\right| \leqq C n^{M} \text { and }\left|b_{\nu}\right| \leqq C \nu^{M}
$$

hold.

Condition (14) guarantees that the series $Z_{A}(s) \underset{\text { def }}{=} \sum_{n=1}^{\infty} a_{n} / n^{s}$ and $Z_{B}(s) \underset{\text { def }}{=} \sum_{\nu=1}^{\infty} b_{\nu} / \nu^{s}$ both converge on the domain $\{s \in \mathbb{C} ; \operatorname{Re} s>M\}$. Furthermore it is clear that they are bounded on $\{s \in \mathbb{C} ; \operatorname{Re} s \geqq M+\varepsilon\} \quad(\varepsilon$ $>0)$. We now suppose that $Z_{A}(s)$ and $Z_{B}(s)$ can be analytically continued to the whole $s$-plane as meromorphic functions having their poles only at $s=1$. We denote thus extended functions again by $Z_{A}(s)$ and $Z_{B}(s)$, respectively. We further assume that $(s-1) Z_{A}(s)$ and $(s-1)$ $Z_{B}(s)$ are entire functions of finite order.

Now our result corresponding to the classical result of Hamburger is the following:

Theorem 2. Suppose that $Z_{A}(s)$ and $Z_{B}(s)$ satisfy

$$
\frac{\Gamma(s / 2)}{\pi^{s / 2}} Z_{A}(s)=\frac{\Gamma((1-s) / 2)}{\pi^{(1-s) / 2}} Z_{B}(1-s)
$$

Then $Z_{A}(s)=Z_{B}(s)$ and it is a constant multiple of the zetafunction $\zeta(s)=\sum_{n=1}^{\infty} 1 / n^{s}$ of Riemann.

Proof. Following Sato [6] we first define the following integral $I_{A}(s, \varphi)$ for $\varphi$ in the Schwartz space $\mathscr{S}$ when $\operatorname{Re} s>M$.

$$
I_{A}(s, \varphi)=\int_{0}^{\infty} t^{s-1}\left(\sum_{n \in \mathbb{Z}^{+}} a_{n} \varphi(t n)+\sum_{n \in \mathbf{Z}^{-}} a_{-n} \varphi(t n)\right) d t
$$

Here and in what follows $\mathbb{Z}^{+}$(resp., $\mathbb{Z}^{-}$) denotes the set of strictly positive (resp., strictly negative) integers. Similarly we define $J_{B}(s, \varphi)$ by

$$
\int_{0}^{\infty} t^{-s}\left(\sum_{\nu \in \mathbf{Z}^{+}} b_{\nu} \widehat{\varphi}(t \nu)+\sum_{\nu \in \mathbf{Z}^{-}} b_{-\nu} \widehat{\varphi}(t \nu)\right) d t
$$

on the domain $\{s \in \mathbb{C} ; \operatorname{Re} s<1-M\}$. Since $\varphi$ is in $\mathscr{S}$, the condition (14) guarantees that $I_{A}(s, \varphi)$ and $J_{B}(s, \varphi)$ are well-defind when $\operatorname{Re} s$ (resp., $-\operatorname{Re} s$ ) is sufficiently large. Furthermore we can easily verify that

$$
I_{A}(s, \varphi)=Z_{A}(s) \int_{-\infty}^{\infty}|x|^{s-1} \varphi(x) d x
$$


holds on $\{s \in \mathbb{C} ; \operatorname{Re} s>M+1\}$ and that

$$
J_{B}(s, \varphi)=Z_{B}(1-s) \int_{-\infty}^{\infty}|\xi|^{-s} \widehat{\varphi}(\xi) d \xi
$$

holds on $\{s \in \mathbb{C} ; \operatorname{Re} s<-M\}$.

Integration by parts (see Gel'fand-Shilov [2], p. 90) shows that $\int_{-\infty}^{\infty}|x|^{s-1} \varphi(x) d s$ and $\int_{-\infty}^{\infty}|\xi|^{-s} \hat{\varphi}(\xi) d \xi$ are meromorphic functions of $s$; thus $I_{A}(s, \varphi)$ and $J_{B}(s, \varphi)$ are also meromorphic functions of $s$. Furthermore, in view of

$$
\mathscr{L}\left(|\xi|^{-s}\right)=\frac{\pi^{s-1 / 2} \Gamma((1-s) / 2)}{\Gamma(s / 2)}|x|^{s-1},
$$

we conclude from (15) that

$$
I_{A}(s, \varphi)=J_{B}(s, \varphi)
$$

holds on the entire $s$-plane.

In passing, we note that there exist some constants $C_{0}$ and $M_{0}$ such that

$$
\left|\frac{\Gamma((1-s) / 2)}{\Gamma(s / 2)}\right| \leqq C_{0}|\operatorname{Im} s|^{M_{0}}
$$

on $\{s \in \mathbb{C} ;-1-M \leqq \operatorname{Re} s \leqq M+2,|\operatorname{Im} s| \geqq 1\}$. Since $(s-1) Z_{A}(s)$ and $(s-1) Z_{B}(s)$ are of finite order by assumption, it follows from the Phragmén-Lindelöf theorem that (15) and (20) imply that

$$
\left|Z_{A}(s)\right|,\left|Z_{B}(s)\right| \leqq C^{\prime}|\operatorname{Im} s|^{M{ }^{\prime}}
$$

holds on $\{s \in \mathbb{C} ;-1-M \leqq \operatorname{Re} s \leqq M+2,|\operatorname{Im} s| \geqq 1\}$ for some constants $C^{\prime}$ and $M^{\prime}$. On the other hand,

$$
D_{x}^{L}|x|^{s+L-1}=\prod_{l=0}^{L-1}(s+l)|x|^{s-1}
$$

holds on $\{s \in \mathbb{C}$; $\operatorname{Re} s>1-L\}$, we have

$$
\int_{-\infty}^{\infty}|x|^{s-1} \varphi(x) d x=\frac{1}{\prod_{l=0}^{L-1}(s+l)} \int_{-\infty}^{\infty}|x|^{s+L-1}\left(-D_{x}\right)^{L} \varphi(x) d x
$$

there. Hence, by choosing $L$ sufficiently large, we conclude from (22) that 


$$
\left.\left.\left|\int_{-\infty}^{\infty}\right| x\right|^{s-1} \varphi(x) d x\left|\leqq C_{\varphi}\right| \operatorname{Im} s\right|^{-M^{\prime}-1}
$$

holds on the domain $\{s \in \mathbb{C} ;-1-M \leqq \operatorname{Re} s \leqq M+2,|\operatorname{Im} s| \geqq 1\}$ for some constant $C_{\varphi}$ depending on $\varphi$. Therefore (21) combined with (23) enables us to apply Cauchy's formula to $I_{A}(s, \varphi) t^{-s}(t>0)$ to obtain

$$
\begin{aligned}
& \frac{1}{2 \pi \sqrt{-1}} \int_{-1-M-\sqrt{-1} \infty}^{-1-M+\sqrt{-1} \infty} I_{A}(s, \varphi) t^{-s} d s \\
& =\frac{1}{2 \pi \sqrt{-1}} \int_{M+2-\sqrt{-1} \infty}^{M+2+\sqrt{-1} \infty} I_{A}(s, \varphi) t^{-s} d s+\operatorname{Res}_{s \in P(A)}\left[I_{A}(s, \varphi) t^{-s}\right],
\end{aligned}
$$

where $P(A)$ denotes the set of poles of $I_{A}(s, \varphi) t^{-s}$ in the domain $\{s \in \mathbb{C}$; $-1-M<\operatorname{Re} s<M+2\}$. Here we may assume that $I_{A}(s, \varphi) t^{-s}$ has no poles either on the line $\{s \in \mathbb{C} ; \operatorname{Re} s=-1-M\}$ or on the line $\{s \in \mathbb{C}$; $\operatorname{Re} s=M+2\}$ by changing $M$ slightly, if necessary. Since $\int_{-\infty}^{\infty}|x|^{s-1} \varphi(x) d x$ has its poles only at $s=0,-2,-4, \cdots$ (Gel'fand-Schilov [2], p. 90), and since $Z_{A}(s)$ is supposed to have its pole only at $s=1, P(A)$ consists of $1,0,-2, \cdots,-2 p$ with $p=\max \{q \in \mathbb{Z} ;-1-M<-2 q\}$. Note that $t^{-s}$ is an entire function of $s$, if $t>0$. Since the residue of the distribution $|x|^{s-1}$ at $s=2 q$ is known to be $2 D_{x}^{2 q} \delta(x) /(2 q)$ ! (Gel'fandShilov [2], p. 91), (19) and (24) imply

$$
\begin{aligned}
& \frac{1}{2 \pi \sqrt{-1}} \int_{-1-M-\sqrt{-1} \infty}^{-1-M+\sqrt{-1} \infty} J_{B}(s, \varphi) t^{-s} d s \\
& =\frac{1}{2 \pi \sqrt{-1}} \int_{M+2-\sqrt{-1} \infty}^{M+2+\sqrt{-1} \infty} I_{A}(s, \varphi) t^{-s} d s+B(t) \int_{-\infty}^{\infty} \varphi(x) d x \\
& \quad+\sum_{q=0}^{p} \frac{2 A_{2 q}(t)}{(2 q) !} \int_{-\infty}^{\infty}\left(D_{x}^{2 q} \delta(x)\right) \varphi(x) d x,
\end{aligned}
$$

where $B(t)$ is $\operatorname{Res}_{s=1}\left(Z_{A}(s)\right) / t$ and $A_{2 q}(t)=Z_{A}(2 q) t^{-2 q}$. By the theory of Mellin transformation we obtain from (25)

$$
\begin{aligned}
& t^{-1}\left(\sum_{\nu \in \mathbb{Z}^{+}} b_{\nu} \widehat{\varphi}(\nu / t)+\sum_{\nu \in \mathbb{Z}^{-}} b_{-\nu} \widehat{\varphi}(\nu / t)\right) \\
& =\sum_{n \in \mathbb{Z}^{+}} a_{n} \varphi(t n)+\sum_{n \in \mathbb{Z}^{-}} b_{-n} \varphi(t n)+B(t) \widehat{\varphi}(0) \\
& \quad+\sum_{q=0}^{p} \frac{2 A_{2 q}(t)}{(2 q) !} \int_{-\infty}^{\infty}\left(D_{x}^{2 q} \delta(x)\right) \varphi(x) d x .
\end{aligned}
$$

Here we have used the fact that the equality 


$$
J_{B}(s, \varphi)=\int_{0}^{\infty} t^{s-2}\left(\sum_{\nu \in \mathbf{Z}^{+}} b_{\nu} \widehat{\varphi}(\nu / t)+\sum_{\nu \in \mathbf{Z}^{-}} b_{-\nu} \widehat{\varphi}(\nu / t)\right) d t
$$

holds by changing $t$ to $1 / t$. Setting $t=1$ in (26), we have

$$
\begin{aligned}
\mathscr{I} & \left(\sum_{\nu \in \mathbf{Z}^{+}} b_{\nu} \delta(\xi-\nu)-B(1) \delta(\xi)+\sum_{\nu \in \mathbb{Z}^{-}} b_{-\nu} \delta(\xi-\nu)\right) \\
& =\sum_{n \in \mathbf{Z}^{+}} a_{n} \delta(x-n)+\sum_{n \in \mathbf{Z}^{-}} a_{-n} \delta(x-n)+\sum_{q=0}^{p} \frac{2 A_{2 q}(1)}{(2 q) !} D_{x}^{2 q} \delta(x) .
\end{aligned}
$$

Then it follows from Theorem 1 that the right hand side of (27) has the form $P\left(x, D_{x}\right)\left(\sum_{n \in Z} \delta(x-n)\right)$ for some linear differential operator with polynomial coefficients $P\left(x, D_{x}\right)$. If we rewrite $P$ in the form $Q\left(D_{x}, x\right),{ }^{(*)}$ then the order $m$ of $P$ is the same as that of $Q$. Suppose that $m$ were not zero. It follows from the definition of $Q$ that

$$
P\left(x, D_{x}\right)\left(\sum_{n \in \boldsymbol{Z}} \delta(x-n)\right)=\sum_{n \in \boldsymbol{Z}} Q\left(D_{x}, n\right) \delta(x-n)
$$

holds. On the other hand, the right hand side of (27) contains no derivatives of $\delta(x-n)$ if $n \neq 0$. Since the $m$-th order term of $Q$ has the form $D_{x}^{m} \cdot q_{m}(x)$ with a polynomial $q_{m}(x)$, this means that $q_{m}(n)=0$ shold hold for $n \neq 0$. Hence the polynomial $q_{m}(x)$ should be zero. This is a contradiction. Therefore $P\left(x, D_{x}\right)$ is of the form $P(x)$, where $P(x)$ is a polynomial. Hence $A_{2 q}(1)$ must be zero if $q \neq 0.0^{* *}$ Since no derivative of $\delta(\xi-\nu)$ is contained in the inverse Fourier transform of the left hand side of (27), a special case $(m=N=1)$ of Theorem 1 asserts that $P(x)$ is a constant. Therefore there exists a constant $c$ such that $a_{n}=c$ and $b_{\nu}=c$ hold for every $n$ and $\nu$ in $Z^{+}$. This means that $Z_{A}(s)=Z_{B}(s)$ $=c \zeta(s)$ holds.

Q.E.D.

Next we show a generalization of Hamburger's result to the zeta function associated to $\boldsymbol{Q}(\sqrt{-1})$. In this case we consider not only the usual zeta function but also the zeta function with Grössencharacter.

Let $\left\{a_{m, n}\right\}_{(m, n) \in \boldsymbol{Z}^{2}-\{0\}}$ and $\left\{b_{\mu, \nu}\right\}_{(\mu, \nu) \in \boldsymbol{Z}^{2-\{0\}}}$ be sets of complex numbers

(*) This means that the multiplication by $x$ is applied first and then comes the differentiation.

(**) This fact can be directly verified by observing that the poles of $J_{B}(s, \varphi)$ are confined to $\{s=0,1,3,5, \cdots\}$. See the proof of Theorem 3 below, where we will use the reasoning of this type. 
which satisfy the condition (28) below.

(28) There exist constants $C$ and $C^{\prime}$ for which

$$
\left|a_{m, n}\right| \leqq C\left(m^{2}+n^{2}\right)^{C^{\prime}} \text { and }\left|b_{\mu, \nu}\right| \leqq C\left(\mu^{2}+\nu^{2}\right)^{C^{\prime}}
$$

hold.

This condition guarantees that the series

$$
Z_{\sqrt{-1}, A}^{l}(s) \underset{\operatorname{def}}{=} \sum_{(m, n) \in \mathbb{Z}^{2}-\{0\}} a_{m, n} \frac{\exp (\sqrt{-1} l \arg (m+\sqrt{-1} n))}{\left(m^{2}+n^{2}\right)^{s}}
$$

and

$$
Z_{\sqrt{-1, B}}^{l}(s) \underset{\text { def }}{=} \sum_{(\mu, \nu) \in Z^{2}-\{0\}} b_{\mu, \nu} \frac{\exp (\sqrt{-1} l \arg (\mu+\sqrt{-1} \nu))}{\left(\mu^{2}+\nu^{2}\right)^{s}}
$$

converge on the domain $\left\{s \in \mathbb{C} ; \operatorname{Re} s>C^{\prime}\right\}$ for every integer $l$. We now suppose that $(s-1) Z_{\sqrt{-1}, A}^{0}(s), \quad(s-1) Z_{\sqrt{-1}, B}^{0}(s), \quad Z_{\sqrt{-1, A}}^{l}(s) \quad(l \neq 0)$ and $Z_{\sqrt{-1, B}}^{l}(s) \quad(l \neq 0)$ can be analytically continued to the whole $s$-plane as entire functions of finite order. We denote these extended functions again by $Z_{\sqrt{-1}, A}^{l}(s)$ and $Z_{\sqrt{-1}, B}^{l}(s)$, respectively. Then we have the following generalization of Hamburger's result.

Theorem 3. Suppose that $Z_{\sqrt{-1}, A}^{l}(s)$ and $Z_{\sqrt{-1}, B}^{l}(s)$ satisfy

$$
\frac{\Gamma\left(s-\frac{l}{2}\right) Z_{\sqrt{-1}, A}^{l}(s)}{\pi^{s}}=\frac{\exp (\sqrt{-1} l \pi / 2) \Gamma\left(1-s-\frac{l}{2}\right) Z_{\sqrt{-1, B}}^{l}(1-s)}{\pi^{1-s}}
$$

Then $Z_{\sqrt{-1, A}}^{l}(s)=Z_{\sqrt{-1}, B}^{l}(s)$ holds and there exists a constant $c$ such that it is equal to $c \zeta_{\sqrt{-1}}^{l}(s)$, where

$$
\zeta_{\sqrt{-1}}^{l}(s)=\sum_{(m, n) \in \mathbf{Z}^{2}-\{0\}} \frac{\exp (\sqrt{-1} l \arg (m+\sqrt{-1} n))}{\left(m^{2}+n^{2}\right)^{s}} .
$$

Proof. We first define the following integral $I_{A}^{l}(s, \varphi)$ for $\varphi$ in $\mathscr{S}_{\mathbb{R}^{2} \text {, }}$ when $\operatorname{Re} s>C^{\prime}$.

$$
\begin{aligned}
& I_{A}^{l}(s, \varphi) \\
= & \int_{0}^{2 \pi}\left(\int_{0}^{\infty}\left(\sum_{(m, n) \in \mathbb{Z}^{2}-\{0\}} a_{m, n} \varphi(m t \cos \theta-n t \sin \theta, m t \sin \theta+n t \cos \theta)\right) \times\right.
\end{aligned}
$$




$$
\left.\times \exp (-\sqrt{-1} l \theta) t^{2 s-1} d t\right) d \theta
$$

For the simplicity of notations, we denote $\cos \theta+\sqrt{-1} \sin \theta$ and $m+\sqrt{-1}$ n by $\Theta$ and $M$, respectively. Similary, let $J_{B}(s, \varphi)$ denote

$$
\begin{aligned}
\int_{0}^{2 \pi}\left(\int _ { 0 } ^ { \infty } \left(\sum_{(\mu, \nu) \in \mathbb{Z}^{2}-\{0\}} b_{\mu, \nu} \widehat{\varphi}(\operatorname{Re}(t \widehat{M} \Theta),\right.\right. \\
\left.\operatorname{Im}(t \widehat{M} \Theta))) \exp (-\sqrt{-1} l \theta) t^{-2 s} d t\right) d \theta,
\end{aligned}
$$

where $\widehat{M}$ is, by definition, $\mu+\sqrt{-1} \nu$. Since $\varphi$ is in $\mathscr{S}_{\boldsymbol{R}^{2}}$, the condition (28) guarantees that $J_{B}(s, \varphi)$ is well-defined on the domain $\{s \in \mathbb{C} ; \operatorname{Re} s$ $\left.<-C^{\prime}+1 / 2\right\}$.

It immediately follows from the definition that

$$
\begin{aligned}
I_{A}^{l}(s, \varphi)= & Z_{\sqrt{-1, A}}^{l}(s) \int \varphi(x, y) \times \\
& \times \exp (-\sqrt{-1} l \arg (x+\sqrt{-1} y))\left(x^{2}+y^{2}\right)^{s-1} d x d y
\end{aligned}
$$

and

$$
\begin{aligned}
J_{B}^{l}(s, \varphi)= & Z_{\sqrt{-1}, B}^{l}(1-s) \int \hat{\varphi}(\xi, \eta) \times \\
& \times \exp (-\sqrt{-1} l \arg (\xi+\sqrt{-1} \eta))\left(\xi^{2}+\eta^{2}\right)^{-s} d \xi d \eta
\end{aligned}
$$

hold on their respective domain of convergence. Since $Z_{\sqrt{-1}, A}^{l}(s)$ and $Z_{\sqrt{-1}, B}(s)$ define meromorphic functions of $s, I_{A}^{l}(s, \varphi)$ and $J_{B}^{l}(s, \varphi)$ can be analytically continued to define meromorphic functions of $s$. The thus extended functions are also denoted by $I_{A}^{l}(s, \varphi)$ and $J_{B}^{l}(s, \varphi)$, respectively. Before proceeding further, let us recall the following formula:

Lemma 4. $\mathscr{E}\left(r^{2 \sigma} \exp (\sqrt{-1} l \theta)\right)$

$$
=\pi^{-2 \sigma-1} \frac{\Gamma\left(1+\sigma+\frac{l}{2}\right)}{\Gamma\left(-\sigma+\frac{l}{2}\right)} \rho^{-2-2 \sigma} \exp \left(\sqrt{-1} l\left(\hat{\theta}+\frac{\pi}{2}\right)\right),
$$

where $(r, \theta)$ and $(\rho, \hat{\theta})$ denote respectively the polar coordinate systems of $\boldsymbol{R}_{x, y}^{2}$ and $\boldsymbol{R}_{\xi, \eta}^{2}$, that is, $x=r \cos \theta, y=r \sin \theta, \xi=\rho \cos \hat{\theta}$ and $\eta=\rho \sin \hat{\theta}$. 
This formula follows from the integral representation of the Bessel function $J_{l}(z)$, i.e.,

$$
J_{l}(z)=\frac{1}{2 \pi} \int_{\alpha}^{2 \pi+\alpha} \exp (\sqrt{-1}(l \theta-z \sin \theta)) d \theta
$$

and a formula for Hankel transformation, i.e.,

$$
\begin{aligned}
& \int_{0}^{\infty} x^{\mu} J_{l}(x y)(x y)^{1 / 2} d x \\
& \quad=2^{\mu+1 / 2} y^{-\mu-1} \frac{\Gamma\left(\frac{1}{2} \mu+\frac{1}{2} l+\frac{3}{4}\right)}{\Gamma\left(-\frac{1}{2} \mu+\frac{1}{2} l+\frac{1}{4}\right)} \quad(y>0) .
\end{aligned}
$$

(Bateman Manuscript Project, vol. II [1], p. 22 (7)). Note that the restriction on the parameters given in [1] can be removed by the analytic continuation.

Now, combining the above formula and the assumption (29), we find that

$$
I_{A}^{l}(s, \varphi)=J_{B}^{l}(s, \varphi)
$$

holds on the entire s-plane. Then, proceeding in the same way as in the proof of Theorem 2, we obtain

$$
\begin{aligned}
& \frac{1}{2 \pi \sqrt{-1}} \int_{-1-C^{\prime}-\sqrt{-1} \infty}^{-1-C^{\prime}+\sqrt{-1} \infty} J_{B}^{l}(s, \varphi) t^{-2 s} d s \\
& =\frac{1}{2 \pi \sqrt{-1}} \int_{C^{\prime}+2-\sqrt{-1} \infty}^{C^{\prime}+2+\sqrt{-1} \infty} I_{A}^{l}(s, \varphi) t^{-2 s} d s+\operatorname{Res}_{s \in P(A, l)}\left[I_{A}^{l}(s, \varphi) t^{-s}\right],
\end{aligned}
$$

where $P(A, l)$ denotes the set of poles of $I_{A}^{l}(s, \varphi) t^{-s}(t>0)$ in the domain $\left\{s \in C ;-1-C^{\prime}<\operatorname{Re} s<C^{\prime}+2\right\}$. Here we have assumed that $J_{B}^{l}(s, \varphi) t^{-2 s}$ has no poles on the line $\left\{s \in \mathbb{C} ; \operatorname{Re} s=-1-C^{\prime}\right\}$ and that $I_{A}^{l}(s, \varphi) t^{-2 s}$ has no poles on the line $\left\{s \in \mathbb{C} ; \operatorname{Re} s=C^{\prime}+2\right\}$, which can be achieved by changing $C^{\prime}$ slightly, if necessary. First let us consider the case where $l$ is equal to zero. Then

$$
\int \varphi(x, y)\left(x^{2}+y^{2}\right)^{s-1} d x d y
$$

has a pole when $2 s-1=-(2 p-1)$ holds for a strictly positive integer $p$, i.e., when $s$ is a non-positive integer. Furthermore its residue at $s=0$ 
is known to be $\pi \iint \delta(x) \delta(y) \varphi(x, y) d x d y$. (Gel'fand-Shilov [2], p. 72) Hence the set of poles of $I_{A}^{0}(s, \varphi)$ is contained in $\{s ; s=1,0,-1, \cdots\}$. By the same reasoning we find that the set of poles of $J_{B}^{0}(s, \varphi)$ is contained in $\{s ; s=0,1,2,3, \cdots\}$. Hence it follows from (32) that $I_{A}^{0}(s, \varphi)$ has its poles only at $s=0$ or 1 . Therefore (33) entails

$$
\begin{aligned}
& \frac{1}{2 \pi \sqrt{-1}} \int_{-1-C^{\prime}-\sqrt{-1} \infty}^{-1-C^{\prime}+\sqrt{-1} \infty} J_{B}^{0}(s, \varphi) t^{-2 s} d s \\
& =\frac{1}{2 \pi \sqrt{-1}} \int_{C^{\prime}+2-\sqrt{-1} \infty}^{C^{\prime}+2+\sqrt{-1} \infty} I_{A}^{0}(s, \varphi) t^{-2 s} d s+B(t) \iint \varphi(x, y) d x d y \\
& \quad+A(t) \iint \delta(x) \delta(y) \varphi(x, y) d x d y
\end{aligned}
$$

where $B(t)$ is $\operatorname{Res}_{s=1}\left[Z_{\sqrt{-1}, A}^{0}(s) / t\right]$ and $A(t)=\pi Z_{\sqrt{-1, A}}^{0}(0)$. Proceeding again in the same way as in the proof of Theorem 2 , we obtain

$$
\begin{gathered}
\int_{0}^{2 \pi}\left[\sum_{(\mu, \nu) \in \mathbf{Z}^{2}-\{0\}} b_{\mu, \nu} \widehat{\varphi}(\operatorname{Re}(\widehat{M} \Theta), \operatorname{Im}(\widehat{M} \Theta))\right] d \theta \\
=\int_{0}^{2 \pi}\left[\sum_{(m, n) \in \mathbf{Z}^{2}-\{0\}} a_{m, n} \varphi(\operatorname{Re}(M \Theta), \operatorname{Im}(M \Theta))\right. \\
\quad+B(1) \widehat{\varphi}(0,0)+A(1) \varphi(0,0)] d \theta .
\end{gathered}
$$

The same reasoning works equally well for the case when $l$ is different from zero. As a matter of fact, it immediately follows from the assumption that $P(A, l)$ is void if $l$ is different from zero. Hence we have

$$
\begin{aligned}
\int_{0}^{2 \pi} & {\left[\sum_{(\mu, \nu) \in \mathbb{Z}^{2}-\{0\}} b_{\mu, \nu} \widehat{\varphi}(\operatorname{Re}(\widehat{M} \Theta), \operatorname{Im}(\widehat{M} \Theta)] \exp (-\sqrt{-1 l \theta}) d \theta\right.} \\
= & \int_{0}^{2 \pi}\left[\sum_{(m, n) \in \mathbf{Z}^{2}-\{0\}} a_{m, n} \varphi(\operatorname{Re}(M \Theta), \operatorname{Im}(M \Theta)] \exp (-\sqrt{-1} l \theta) d \theta\right.
\end{aligned}
$$

for any $l \neq 0$. On the other hand

$$
\int_{0}^{2 \pi}(B(1) \widehat{\varphi}(0,0)+A(1) \varphi(0,0)) \exp (-\sqrt{-1} l \theta) d \theta=0
$$

holds if $l$ is different from zero. Thus we obtain

$$
\int_{0}^{2 \pi}\left[\sum_{(\mu, \nu) \in Z^{2}-\{0\}} b_{\mu, \nu} \widehat{\varphi}(\operatorname{Re}(\widehat{M} \Theta), \operatorname{Im}(\widehat{M} \Theta))\right] \exp (-\sqrt{-1} l \theta) d \theta
$$




$$
\begin{aligned}
= & \int_{0}^{2 \pi}\left[\sum_{(m, n) \in \mathbf{Z}^{2}-\{0\}} a_{m, n} \varphi(\operatorname{Re}(M \Theta), \operatorname{Im}(M \Theta))\right. \\
& +B(1) \hat{\varphi}(0,0)+A(1) \varphi(0,0)] \exp (-\sqrt{-1} l \theta) d \theta
\end{aligned}
$$

for every $l$. This implies

$$
\begin{aligned}
& \sum_{(\mu, \nu) \in \mathbb{Z}^{2}-\{0\}} b_{\mu, \nu} \widehat{\varphi}(\operatorname{Re}(\widehat{M} \Theta), \operatorname{Im}(\widehat{M} \Theta)) \\
= & \sum_{(m, n) \in \mathbf{Z}^{2}-\{0\}} a_{m, n} \varphi(\operatorname{Re}(M \Theta), \operatorname{Im}(M \Theta))+B(1) \widehat{\varphi}(0,0)+A(1) \varphi(0,0) .
\end{aligned}
$$

By choosing $\Theta$ to be 1 , we apply Theorem 1 to conclude that

$$
a_{m, n}=b_{\mu, \nu}=A(1)=-B(1)
$$

holds for every $(m, n)$ and $(\mu, \nu)$ in $\mathbb{Z}^{2}-\{0\}$. This is the required result.

Q.E.D.

Remark 1. It would be interesting if one could prove the theorem by assuming the functional equation (29) only for $l=0$. The celebrated result of Hecke [5] asserts such a stronger result on the additional assumption that $Z_{\sqrt{-1, A}}^{0}(s)=Z_{\sqrt{-1}, B}^{0}(s)$. It is strange that Hecke [4] gave a new proof of Hamburger's theorem in which $A$ is not assumed to be equal to $B$, while in his celebrated paper [5] he made no reference to the case $A \neq B$.

Remark 2. It is interesting to compare the results of Theorems 1 and 2 and of Theorems 1 and 3. In Theorem 2 we use Dirichlet series $\sum a_{n} n^{-s}$ with $n \in \mathbb{Z}^{+}$. For this reason we note, by examining the proof of Theorem 2, that we apply only the special case of Theorem 1 for $m=N=1$ but, more important, for sequences $\left\{a_{0, n}\right\},\left\{b_{0, \nu}\right\}$ which are even in $n$ and $\nu$ respectively. We could actually use Hamburger's theorem to derive this special case of Theorem 1, by noting that the linear combinations of the distributions $|x|^{s}$ are dense in the even distributions in $\mathscr{S}^{\prime}$ (if we ignore the point $x=0$ which requires the special consideration given in the proof of Theorem 2).

On the other hand, Theorems 1 and 3 are, essentially, equivalent because we used all the Grössencharacters.

A. Weil [7] has given an extension of Hecke's work [5] in a dif- 
ferent direction. He uses ordinary (congruence) characters instead of Grössencharacters.

\section{Acknowledgment}

The second named author (T.K.) would like to thank Dr. T. Oda for having given him a clear-cut explanation on several questions on $\zeta$-functions and $\theta$-functions. He would like to thank his colleagues at Massachusetts Institute of Technology for their hospitality shown to him during his stay there. This work was completed then under the support of NSF MCS 8006521.

\section{References}

[1] Bateman Manuscript Project (A. Erdélyi et al.), Tables of Integral Transforms, II, New York-Tronto-London, McGraw-Hill, 1954.

[2] Gel'fand, I. M. and Shilov, G. E., Generalized Functions, I, New York-London, Academic Press, 1964. (Russian original: 1958).

[3] Hamburger, H., Über die Riemannsche Funktionalgleichung der $\zeta$-Funktion, Math. Z., 10 (1921), 240-254.

[4] Hecke, E., Über die Lösungen der Riemannschen Funktionalgleichang, Math. Z., 16 (1923), 301-307 (Werke, 374-380).

[5] - Über die Bestimmung Dirichletscher Reihen durch ihre Funktionalgleichung, Math. Ann., 111 (1936), 664-699 (Werke, 591-626).

[6] Sato, M., Theory of pre-homogeneous vector spaces, Sûgaku no Ayumi, 15 (1970), 85-157. (Noted by S. Shintani, in Japanese.)

[7] Weil, A., Über die Bestimmung Dirichletscher Reihen durch Funktionalgleichungen, Math. Ann., 168 (1967), 145-156. 